\title{
Characterization of mixed DODAB/monoolein aggregates using Nile Red as a solvatochromic and anisotropy fluorescent probe
}

\author{
J.P. Neves Silva, M.E.C.D. Real Oliveira, P.J.G. Coutinho* \\ Physics Department, University of Minho, Campus of Gualtar, 4710-057 Braga, Portugal
}

\section{A R T I C L E I N F O}

\section{Article history:}

Received 8 August 2008

Received in revised form 8 December 2008

Accepted 12 December 2008

Available online 24 December 2008

\section{Keywords:}

DODAB

Monoolein

Nile Red

Polarity

Hydration

\begin{abstract}
A B S T R A C T
The aim of this study was to get a further knowledge of the interactions between lamellar and non-lamellar forming-phospholipids, as a tool to better understand the membrane dynamics and function. We have studied the behavior of aqueous mixtures of a cationic lipid (dioctadecyldimethylammonium bromide) and a neutral phospholipid (1-monoolein) in 10-150 $\mu \mathrm{M}$ total lipid concentration range. The effect of 1-monoolein on the physical properties (microviscosity, hydration and polarity) of the self-organized mixed aggregates was accessed by studying the steady-state fluorescence emission and anisotropy of the dye Nile Red. This information was combined with $90^{\circ}$ Static Light Scattering assays in order to detect any structural variations in these vesicle systems. The results were interpreted by the coexistence of vesicular structures and other aggregates probably with a bicontinuous nature.
\end{abstract}

(c) 2009 Elsevier B.V. All rights reserved.

\section{Introduction}

Since its beginning, the study of lipid polymorphism has allowed a better understanding of the physical properties of biological membranes and their influence on normal cell function [1,2]. The first experimental observations in this area showed the existence of some highly complex lipid structures that could not be indexed on any simple lattice, and that were mistakenly interpreted as variations of a lamellar organization [3]. It was not until Luzzati work in the beginning of the 1960s that the occurrence of non-lamellar structures was verified, with the identification of the cubic and hexagonal lyotropic phases (Fig. 1) [3].

The development of NMR, X-ray and electron microscopy techniques soon provided the adequate tools for the characterization of these structures and to the establishment of a correlation between the molecular shape of the surfactant and the type of phase formed $[4,5]$. By then, several physical properties such as elastic deformation [6], bilayer thickness [7], interfacial polarity [8], membrane fluidity [9] and curvature strain [10] were already being investigated under temperature and pressure variations in several lipidic models.

It is well established that dioctadecyldimethylammonium bromide (DODAB) molecules, a synthetic lipid, in the presence of excess water, tends to form large unilamellar vesicles (LUV's). The structural organization of these vesicles depends on the lipid

\footnotetext{
* Corresponding author. Tel.: +351 253604321; fax: +351 253604061 .

E-mail address: pcoutinho@fisica.uminho.pt (P.J.G. Coutinho).
}

concentration, the method of preparation, the solvent composition, the temperature and the presence of other substances [11-16]. Long-chain vesicle-forming surfactants like DODAB exhibit a small critical vesicle concentration (CVC), above which vesicles are formed. The CVC of DODAB is too low to be measured by ordinary techniques (for instance surface tension, conductimetry, light scattering, etc.) [12-16], and allows the formation of cationic aggregates in the domain of micromolar concentrations.

One of the most widely studied lipid amphiphiles is the monoglyceride 1-monoolein ( $\mathrm{MO}$ ), a neutral surfactant that has the particularity of presenting two inverse bicontinuous cubic phases $\left(\mathrm{Q}_{\mathrm{II}}{ }^{\mathrm{G}}\right.$ and $\left.\mathrm{Q}_{\mathrm{II}}{ }^{\mathrm{D}}\right)$ on the high water side of the lamellar $\mathrm{L}_{\alpha}$ phase (Fig. 2) [17]. Because it has been demonstrated that these cubic phases play important roles in many cell processes, such as membrane fusion [18], protein function [19,20] and ultra-structural organization [21], MO has become a preferential model for the study of an extensive range of applications. It is well known the use of MO for the crystallization of proteins [22-27], molecular separation in gel electrophoresis medium [28], and DNA condensation in lipoplexes [29].

The physicochemical clarification of the mechanisms involved in the phase transitions between cubic and lamellar phases and the factors that control them, will help understanding the membrane dynamics and developing other possible applications.

The effect of the interfacial membrane surface charge on the structure and stability of cubic-phase membranes was recently investigated in mixtures of MO and the negatively charged lipid dioleoylphosphatidic acid (DOPA) [30]. Using a small angle 


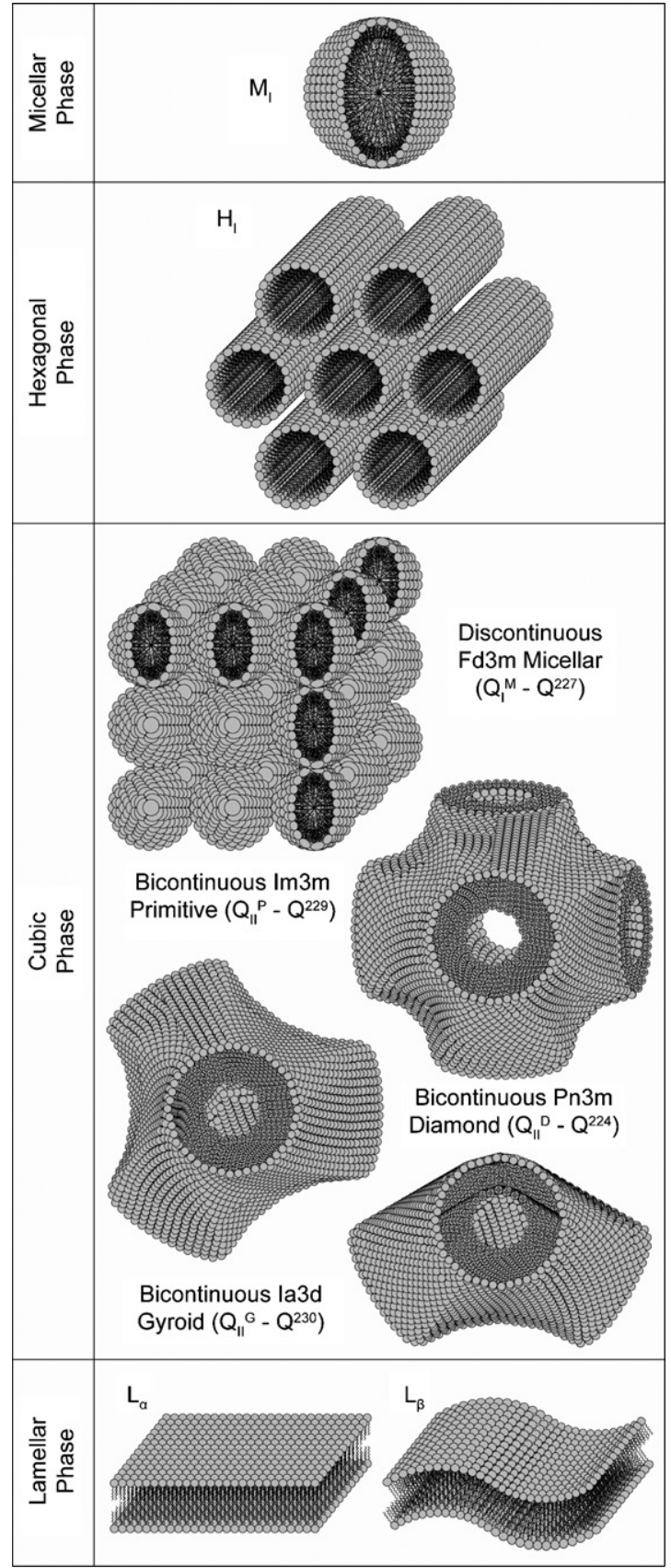

Fig. 1. Representation of some common ordered lyotropic phases of lipids in water including the micellar $(\mathrm{M})$, hexagonal $(\mathrm{H})$, cubic $(\mathrm{Q})$, and lamellar $(\mathrm{L})$ phases.

X-ray scattering (SAXS) technique, it was discovered that an increase in the electrostatic interactions led to a transition $\mathrm{Q}_{\mathrm{II}}{ }^{\mathrm{D}} \rightarrow \mathrm{Q}_{\mathrm{II}}{ }^{\mathrm{P}} \rightarrow \mathrm{L}_{\alpha}$ [30]. Similar approaches, using other negatively charged amphiphiles such as dioleoylphosphatidylserine (DOPS) or dioleoylphosphatidylglycerol (DOPG) corroborated these results, thus indicating surface charge density as an important cubic-tolamellar phase transition control factor [30-36].

In this work, we have decided to study mixtures of MO and the vesicle-forming cationic lipid amphiphile dioctadecyldimethylammonium bromide (DODAB) using Nile Red as a solvatochromic and anisotropy fluorescent probe. The hydrophobic nature of Nile Red and the strong dependence of its steady-state emission properties with the polarity and the hydration level of the medium, substantiate its extensive use on the study of vesicle systems $[35,37]$.

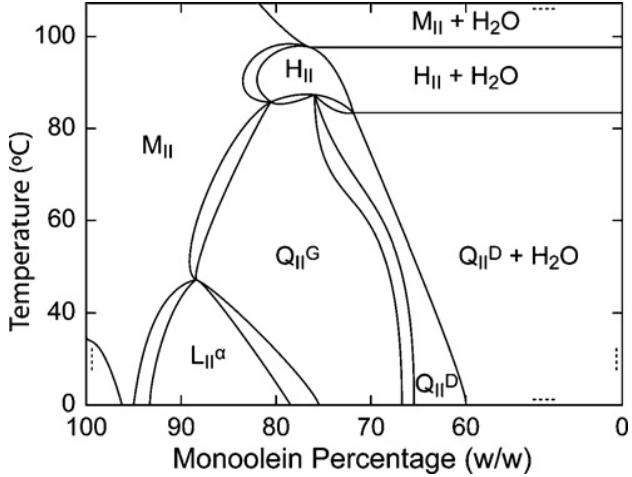

Fig. 2. Monoolein phase diagram presenting two inverse bicontinuous cubic phases $\left(\mathrm{Q}_{\mathrm{II}}{ }^{\mathrm{G}}\right.$ and $\left.\mathrm{Q}_{\mathrm{II}}{ }^{\mathrm{D}}\right)$ on the high water side of the lamellar $\mathrm{L}_{\alpha}$ phase (Fig. 1). Redrawn from Geil et al. [11]

These features enabled us to follow the hydration level and fluidity of DODAB/MO aggregates, and also to detect any structural changes that could indicate the presence of phase transitions due to membrane curvature and interfacial surface charge variation. Further structural information concerning the size of the aggregates was obtained through $90^{\circ}$ Static Light Scattering (SLS) assays.

\section{Materials and methods}

\subsection{Reagents}

The lipid surfactants 1-monoolein (MO) and dioctadecyldimethylammonium bromide (DODAB) were purchased, respectively, from Sigma-Aldrich and Tokyo-Kasei. The solvatochromic and anisotropy probe Nile Red was purchased from Sigma-Aldrich. All reagents were used in the same conditions as received.

\subsection{Liposome preparation and probe resolubilization}

For each liposomal formulation, a predefined volume of Nile Red stock solution in chloroform was transferred to an Eppendorf, so that the final ratio probe/lipid became $1: 500(\mathrm{~mol} / \mathrm{mol})$. After solvent evaporation under a nitrogen steam, the probe film was resolubilized in an appropriate volume of DODAB and MO stock solutions in ethanol $(20 \mathrm{mM})$. The mixture was injected under vigorous vortexing to an aqueous buffer solution at $70^{\circ} \mathrm{C}(30 \mathrm{mM}$, Trizma), so that the final lipid concentration (DODAB + MO) was $1 \mathrm{mM}$ and the different DODAB:MO molar ratios $(1: 2,1: 1$, and $2: 1)$ were obtained.

\subsection{Titration and measurements}

For each experiment, $2500 \mu \mathrm{l}$ of aqueous buffer solution ( $30 \mathrm{mM}$, Trizma) were transferred to a cuvette and titrated with defined volumes of the appropriate liposomal mixtures, until the final lipid concentration was reached. After each addition of cationic liposomes, a 5 min agitation period with a magnetic stirrer was followed, and the polarized emission spectra for Nile Red were then recorded in a Horiba Jobin Yvon Spex Fluorolog-3 spectrofluorimeter using Spex polarizers with $\lambda_{\text {exc }}$ at $525 \mathrm{~nm}$. All spectra were corrected for the instrumental response of the system and the solvent background was subtracted.

The $90^{\circ}$ SLS assays were also performed in the same equipment, with the scattering intensities being recorded in timescans of $60 \mathrm{~s}$ each, with excitation and emission monochromators set respectively to 600 and $601 \mathrm{~nm}$, at which there is neither absorbance, nor fluorescence emission. All the experimental procedures were performed at $25^{\circ} \mathrm{C}$, unless stated otherwise. 


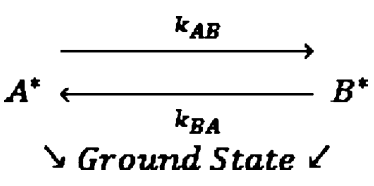

Scheme 1. Two state model for Nile Red fluorescence emission.

\subsection{Nile Red fluorescence emission and anisotropy processing}

A two-state model for excited state behavior of Nile Red involving a solvent relaxation process, where $A^{*}$ and $B^{*}$ represent the initially excited and the relaxed excited state, with the possibility of a reversible reaction (Khrishna model [38]) was assumed (Scheme 1).

The emission spectra of Nile Red were obtained from anisotropy measurements [37]:

$I_{\text {total }}=I_{\mathrm{VV}}+\left(2 \cdot G \cdot I_{\mathrm{VH}}\right)$

The corresponding anisotropy spectrum is given by:

$r(\lambda)=\frac{I_{\mathrm{VV}}-G \cdot I_{\mathrm{VH}}}{I_{\mathrm{VV}}+2 \cdot G \cdot I_{\mathrm{VH}}}$

Both $I_{\mathrm{VV}}$ and $G \cdot I_{\mathrm{VH}}$ were simultaneously fitted to a sum of two lognormal functions [37]:

$$
\begin{aligned}
I_{\mathrm{VV} / \mathrm{VH}}= & \frac{\left(A_{\mathrm{VV} / \mathrm{VH}}\right)_{1}}{\left(\lambda-\left(\lambda_{\max }\right)_{1}+a\right)} \cdot \exp (-c)^{2} \\
& \cdot \exp \left\{-\frac{1}{2 \cdot c^{2}} \cdot\left[\ln \left(\frac{\lambda-\left(\lambda_{\max }\right)_{1}+a}{b}\right)\right]^{2}\right\} \\
& +\frac{\left(A_{\mathrm{VV} / \mathrm{VH}}\right)_{2}}{\left(\lambda-\left(\lambda_{\max }\right)_{2}+a\right)} \cdot \exp (-c)^{2} \\
& \cdot \exp \left\{-\frac{1}{2 \cdot c^{2}} \cdot\left[\ln \left(\frac{\lambda-\left(\lambda_{\max }\right)_{2}+a}{b}\right)\right]^{2}\right\}
\end{aligned}
$$

where $A$ is the maximum emission intensity at $\lambda_{\max }$, and the parameters $a, b$ and $c$ are given by [37]:

$a=H \cdot \frac{\rho}{\rho^{2}-1}$

$b=H \cdot \frac{\rho}{\rho^{2}-1} \cdot \exp \left(c^{2}\right)$

$c=\frac{\ln (\rho)}{\sqrt{2 \cdot \ln (2)}}$

where $H$ and $\rho$ are, respectively, the halfwidth and skewness of the band. Only the parameters with $I_{\mathrm{VV}}$ and $G \cdot I_{\mathrm{VH}}$ subscript depend on whether $I_{\mathrm{VV}}$ or $G \cdot I_{\mathrm{VH}}$ spectra are being fitted. The steady-state fluorescence anisotropies of initially excited state $\left(r_{1}\right)$ and solvent relaxed state $\left(r_{2}\right)$, as well as the emission intensity fraction of the initially excited state $\left(f_{1}\right)$ are given by [37]:

$r_{1}=\frac{\left(A_{\mathrm{VV}}\right)_{1}-\left(A_{\mathrm{VH}}\right)_{1}}{\left(A_{\mathrm{VV}}\right)_{1}+2 \cdot\left(A_{\mathrm{VH}}\right)_{1}}$

$r_{2}=\frac{\left(A_{\mathrm{VV}}\right)_{2}-\left(A_{\mathrm{VH}}\right)_{2}}{\left(A_{\mathrm{VV}}\right)_{2}+2 \cdot\left(A_{\mathrm{VH}}\right)_{2}}$

$f_{1}=\frac{\left(A_{\mathrm{VV}}\right)_{1}+2 \cdot\left(A_{\mathrm{VH}}\right)_{1}}{\left(A_{\mathrm{VV}}\right)_{1}+2 \cdot\left(A_{\mathrm{VH}}\right)_{1}+\left(A_{\mathrm{VV}}\right)_{2}+2 \cdot\left(A_{\mathrm{VH}}\right)_{2}}$

\section{Results and discussion}

Nile Red usually exhibits an increase in fluorescence yield with decreasing solvent polarity, with a corresponding blue shift in the peak emission [38]. In particular, it has been found that fluorescence lifetime of Nile Red markedly decreases with the increase of

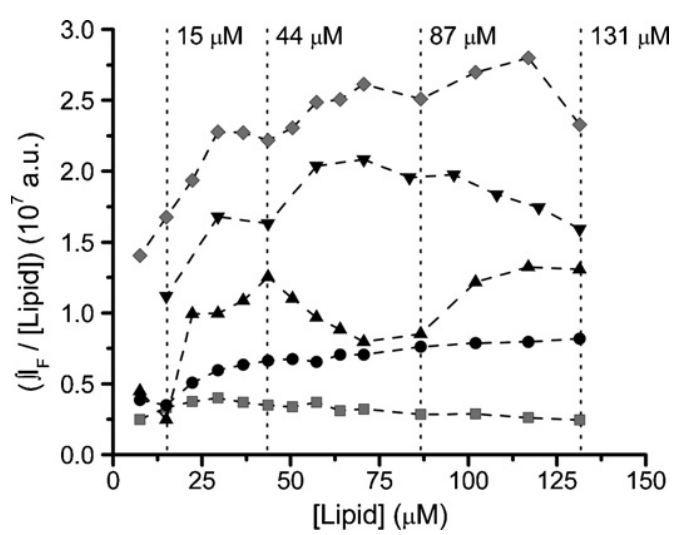

Fig. 3. Variation of Nile Red fluorescence intensity with total lipid concentration

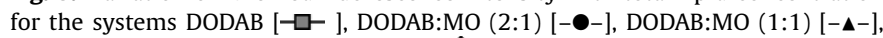
DODAB:MO $(1: 2)[-\nabla-]$ and monoolein $[\checkmark-$ ].

H-bonding capability of the medium [39], and thus can report on the level of hydration of the membrane. Fig. 3 shows the total fluorescence intensity of Nile Red in pure DODAB, pure MO and for three different DODAB:MO molar ratio $(2: 1,1: 1$ and $1: 2)$ at $25^{\circ} \mathrm{C}$. Because the molar ratio between Nile Red and total lipid content was held constant at a value of $1 / 500(\mathrm{~mol} / \mathrm{mol})$ during all titrations, Nile Red fluorescence was expected to be constant, when divided by concentration (Fig. 3). Size and shape characteristics determine light scattering intensity. Thus, in the absence of significant structural changes in the lipid aggregates, $90^{\circ}$ SLS was also expected to be constant when divided by total lipid content (Fig. 4). However, variations in both Nile Red fluorescence intensity and $90^{\circ}$ SLS were observed that can only be caused by structural changes of the aggregates with the total lipid concentration and DODAB:MO molar ratio that results in differences in membrane polarity/hydration felt by Nile Red. There is a general trend for increased fluorescence intensity with the monoolein content and a very different behavior is observed in the $1: 1$ system.

According to the fluorescence variations observed in the $1: 1$ system, we have chosen four specific lipid concentrations (Fig. 3). For pure MO and pure DODAB (in the gel and liquid crystalline phase) the Nile Red fluorescence intensity slightly increases from 15 to $44 \mu \mathrm{M}$ remaining constant in the rest of the titration. For DODAB:MO (2:1), the Nile Red fluorescence intensity follows the same trend than pure MO and pure DODAB, but being lower compared with pure MO, and higher compared with pure DODAB. For DODAB:MO (1:1), the Nile Red fluorescence intensity first increases for lipid concentrations below $44 \mu \mathrm{M}$ and then rapidly decreases

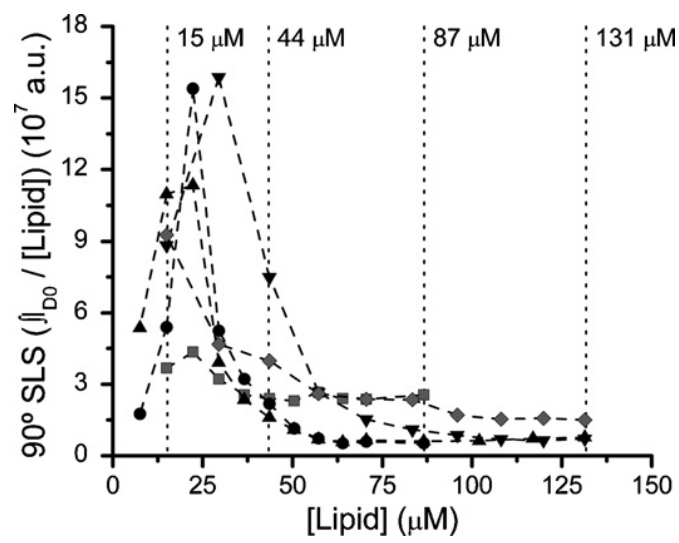

Fig. 4. Variation of $90^{\circ}$ Static Light Scattering (SLS) with total lipid concentration

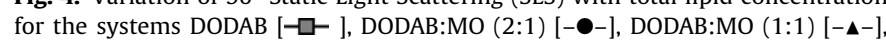
DODAB:MO (1:2) [-7-] and monoolein $[\curvearrowright-]$. 

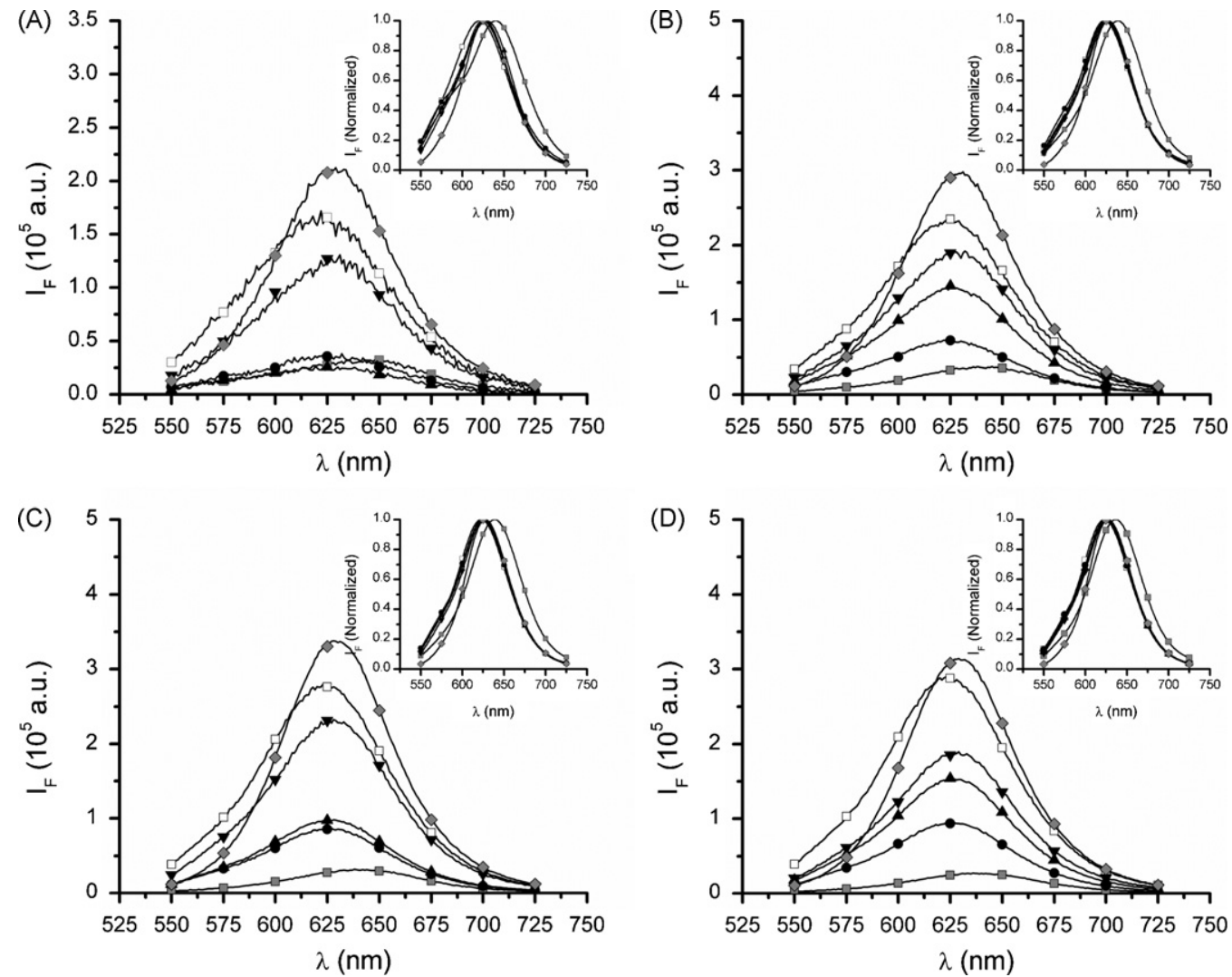

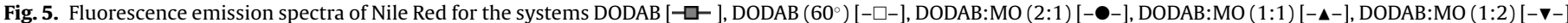
and monoolein $[\checkmark-$ ], at four different concentrations (A: $15 \mu \mathrm{M} ; \mathrm{B}: 44 \mu \mathrm{M} ; \mathrm{C}: 87 \mu \mathrm{M}$; and D: $131 \mu \mathrm{M}$ ).

until $87 \mu \mathrm{M}$, following a new increase in the fluorescence intensity until the end of the titration $(131 \mu \mathrm{m})$. Finally, for DODAB:MO (1:2), the Nile Red fluorescence intensity increases approximately until concentration $87 \mu \mathrm{M}$, following a decrease until the end of the titration.

The $90^{\circ}$ Static Light Scattering assays show a highly dispersive peaked region in the range $0-50 \mu \mathrm{M}$, which is broader for DODAB:MO (1:2). The shape and size seem to stabilize at $60 \mu \mathrm{M}$ for DODAB:MO (1:1 and 2:1), but only at $120 \mu \mathrm{M}$, when monoolein is in excess (DODAB:MO (1:2)). The large aggregates in the DODAB:MO $(1: 1)$ system seem to be more compact than the other cases. The pure MO system shows an intermediate dispersion that decreases with total lipid concentrations. This indicates that the MO structures are becoming more compact. Pure DODAB shows the same peaked behavior but with much less scattering intensity.

Fig. 5 shows the variation in Nile Red fluorescence emission in DODAB:MO systems with different molar ratios (2:1, 1:1 and 1:2) and pure DODAB vesicles in the gel $\left(25^{\circ} \mathrm{C}\right)$ and liquid-crystalline phase $\left(60^{\circ} \mathrm{C}\right)$. There is no spectral shift observed among the three DODAB:MO molar fractions, but only the appearance of a shoulder on the blue side of the emission spectra of Nile Red. Comparing with pure DODAB (gel phase), the presence of monoolein induces a blue shift in the emission spectra of Nile Red. This shift is slightly less pronounced than that observed when pure DODAB changes from the gel to the liquid-crystalline phase. The blue shoulder appears in pure DODAB in gel phase but not in pure MO and in pure DODAB in the liquid-crystalline phase. The change in the spectral shape of Nile Red is more visible at lower lipid concentration. In the mixed systems, the shoulder magnitude on the blue side of the Nile Red spectra for this lipid concentration region follows the order $1: 2<1: 1<2: 1$, corresponding to the order of DODAB content. Above $87 \mu \mathrm{M}$, the spectral shape is constant with total lipid concentration and the blue shoulder is almost lost. Also, it is important to mention that, from 15 to $44 \mu \mathrm{M}$, the blue shoulder in pure DODAB in the gel phase disappears.

Nile Red feels the gel to liquid crystalline transition in DODAB by a blue shift and a huge increase in fluorescence intensity (Fig. 5). As membrane hydration increases with this phase transition, this can only be explained by a relocalization of Nile Red molecules more to the interior of the membrane. The prominence of the blue shoulder for DODAB in the gel phase loses importance as the total lipid concentration increases. Comparing with the spectral changes observed in the mixed DODAB:MO systems, we can conclude that there is no re-localization of Nile Red molecules associated to the structural changes caused by the increase of total lipid concentration and the variation of DODAB to MO molar ratio. We can also conclude that the environment felt by Nile Red molecules in mixed DODAB:MO systems is similar to that reported in DODAB in the liquid crystalline fluid phase $\left(T=60^{\circ} \mathrm{C}\right)$, with spectral variations in the blue side of the spectrum that can be attributed to changes in lipid aggregate structure.

In order to gain further insight on the type of structural changes that are effective upon $\mathrm{MO}$ addition and variation of total lipid concentration we have also studied Nile Red fluorescence anisotropy behavior in DODAB:MO mixed systems.

Fluorescence anisotropy can give information on the microviscosity/fluidity of the membrane. The use of a two state model gives more localized information through Nile Red solvation cage dynamics.

As already mentioned in Section 2.4, this two state model involves a solvent relaxed excited state $\left(B^{*}\right)$ and an initial excited state $\left(A^{*}\right)$, but each one of these states can reflect a superposition of different environments and structures. Fig. 6 illustrates, for the $1: 1$ system, the results of Nile Red fluorescence anisotropy modelization, showing the recovered two components with distinct anisotropies $\left(r_{1}\right.$ and $r_{2}$ ). The corresponding variation of the overall 

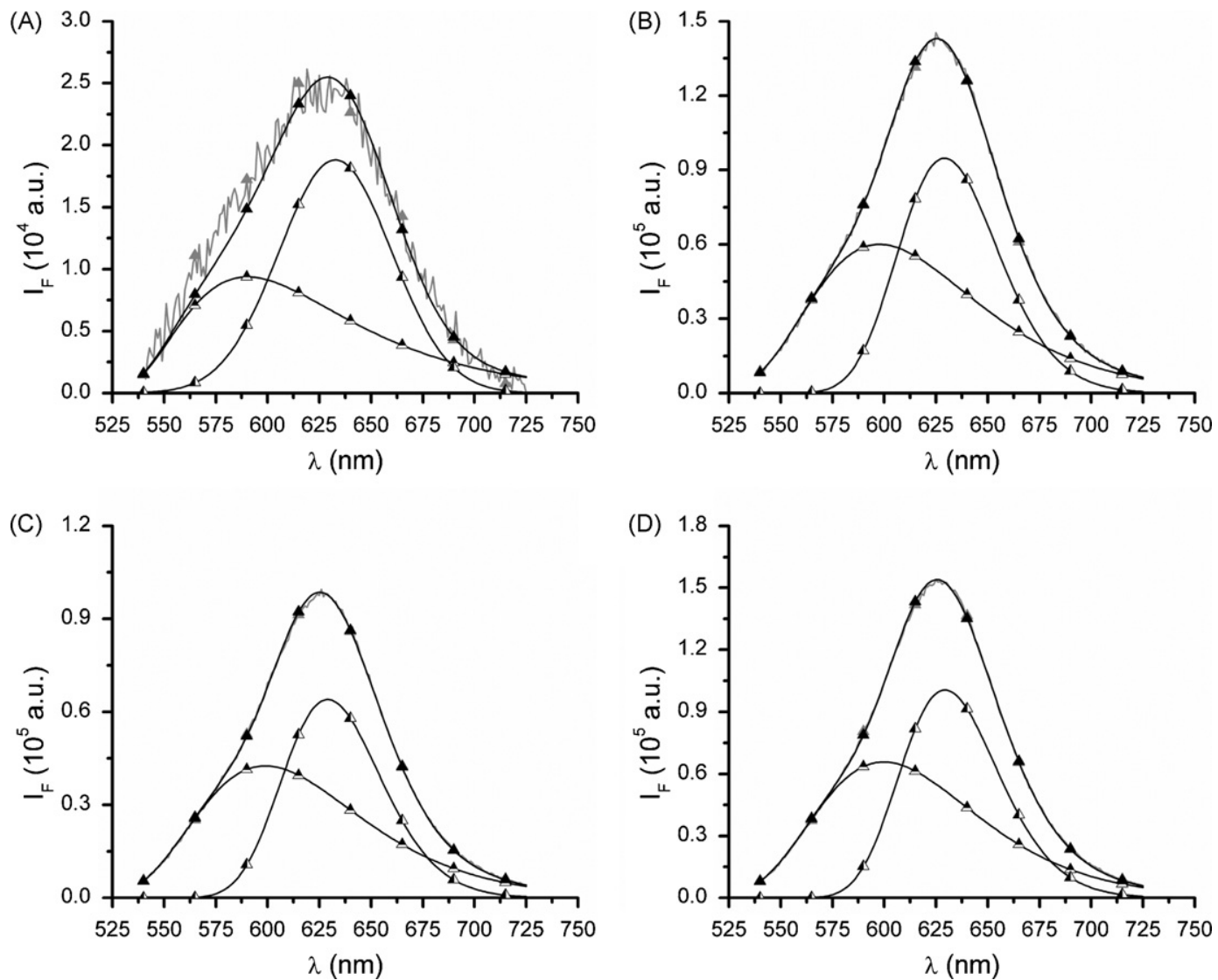

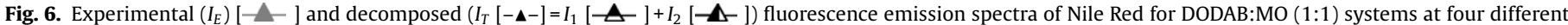

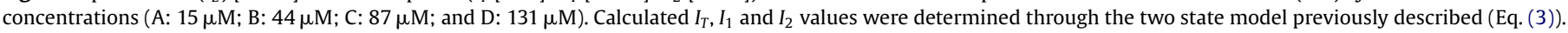

anisotropy $(r)$ with wavelength is shown in Fig. 7. A good correspondence between the experimental results and theoretical model was obtained. Fig. 6 shows that the weight of the components, as well as its spectral positions, depends on the total lipid content, especially in the low concentration region. Also different variations of the overall anisotropy with wavelength are evidenced in Fig. 7.

Fig. 8 compares pure DODAB vesicles in the gel and liquidcrystalline phases, with 100\% MO and the DODAB:MO studied systems. We can see that MO disturbs the ordered gel phase leading to a decrease in membrane fluidity confirming the previous interpretation of the fluorescence spectral features of Nile Red in these systems. In general, the same type of dependence of fluorescence anisotropy with wavelength is observed in pure DODAB, pure $\mathrm{MO}$ and in DODAB:MO. But a closer look shows different behavior depending on MO content and on total lipid concentration. Again it is noticeable a distinct behavior at low total lipid concentration.

In Figs. 9-11, the recovered values for $\lambda_{1}, \lambda_{2}, r_{1}, r_{2}$ and $f_{1}$ are plotted as a function of total lipid concentration. The values for $r_{1}$ and $r_{2}$ for pure MO are lower compared with the values for pure DODAB (gel phase). In the case of DODAB:MO (2:1 and 1:1), the decrease in the anisotropy of the relaxed state $\left(r_{2}\right)$ corresponds to the initial rise of $90^{\circ}$ SLS signal and to the shoulder enhancement of the blue side of the fluorescence spectrum (Fig. 5), which is supported by the variation of the wavelength maximum in the initial excited component. But, when the $90^{\circ}$ SLS decreases, the anisotropy remains constant, suggesting strong structural changes that do not affect the excited state relaxation dynamics of Nile Red. Also pure DODAB in the gel phase shows these same trends but with a much less pronounced peak in $90^{\circ} \mathrm{SLS}$. When monoolein dominates DODAB:MO $(1: 2)$ there is an absence of the blue shoulder and of the $r_{2}$ decrease. However, $90^{\circ}$ SLS shows a similar, but broader, peaked behavior.
In terms of Nile Red excited state relaxation dynamics $\left(r_{1}, r_{2}\right.$ and $f_{1}$ ), the mixed systems shows similar behavior with pure DODAB in the gel phase unless for the $f_{1}$ parameter. This quantity is determined by the relative amounts of the initially excited and the solvent relaxed states. Consequently, it reflects the speed of solvent relaxation when compared to the excited state lifetime.

Above $25 \mu \mathrm{M}$, pure DODAB and pure MO shows a faster relaxation (less initial excited state) than in mixed systems. But in the low concentration range DODAB:MO 2:1 shows a very distinct behavior. The maximum emission wavelength of the initially excited state $\left(\lambda_{1}\right)$ shows an initial red shift with increasing total lipid concentration followed by a stabilization. This variations are consistent with the trends shown by the blue side of the emission spectra shown in Fig. 5, and are leveled off by the presence of excess MO. The maximum emission wavelength of the relaxed excited state $\left(\lambda_{2}\right)$ shows little variation with total lipid concentration with pure DODAB in the gel phase being a remarkable exception with a $15 \mathrm{~nm}$ decrease in the low concentration range $(<25 \mu \mathrm{M})$.

Considering the experimental preparation protocol (Sections 2.2 and 2.3) all samples are prepared by a dilution of a concentrated aqueous lipid solution prepared by injection of an ethanolic lipid mixture in buffer. Thus, the lipid aggregates in the concentrated solutions are expected to be of vesicular structure as mixtures of MO with other lipids with positive spontaneous curvature have a tendency to form lamellar structures by destabilization of cubic phases [30]. From our experimental results we can conclude that the lipid vesicular aggregates changes structure upon dilution, even in the case of pure DODAB. These changes manifest itself as variations of hydration/polarity, microviscosity/fluidity and "solvent" cage dynamics felt by Nile Red fluorescence probe, with corresponding significant size/shape variations seen from Static Light 

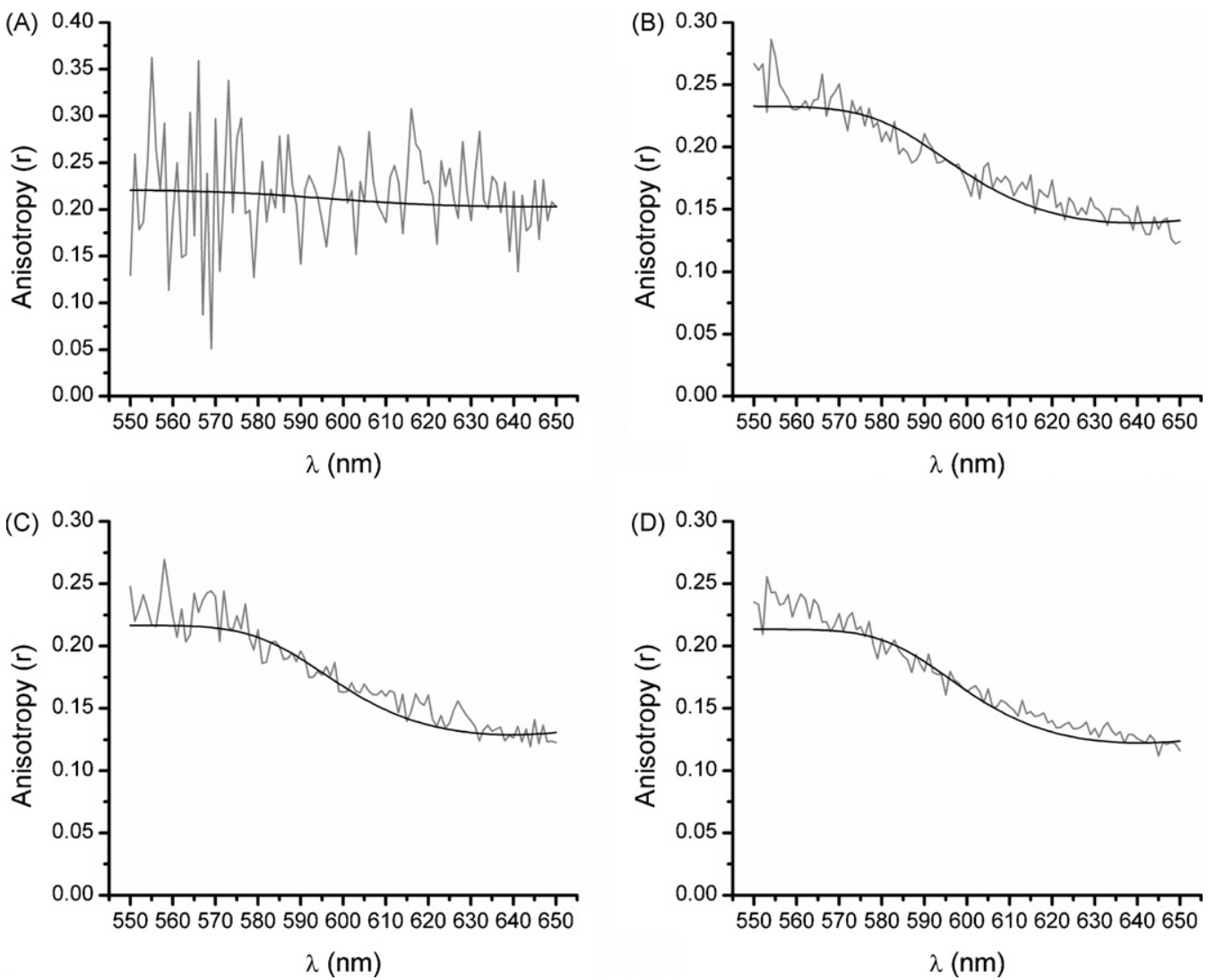

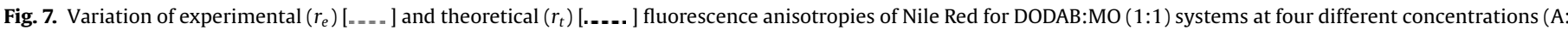
$15 \mu \mathrm{M}$; B: $44 \mu \mathrm{M}$; C: $87 \mu \mathrm{M}$; and D: $131 \mu \mathrm{M}$ ). Calculated $r_{t}$ values were determined through the two state model previously described (Eq. (2)).
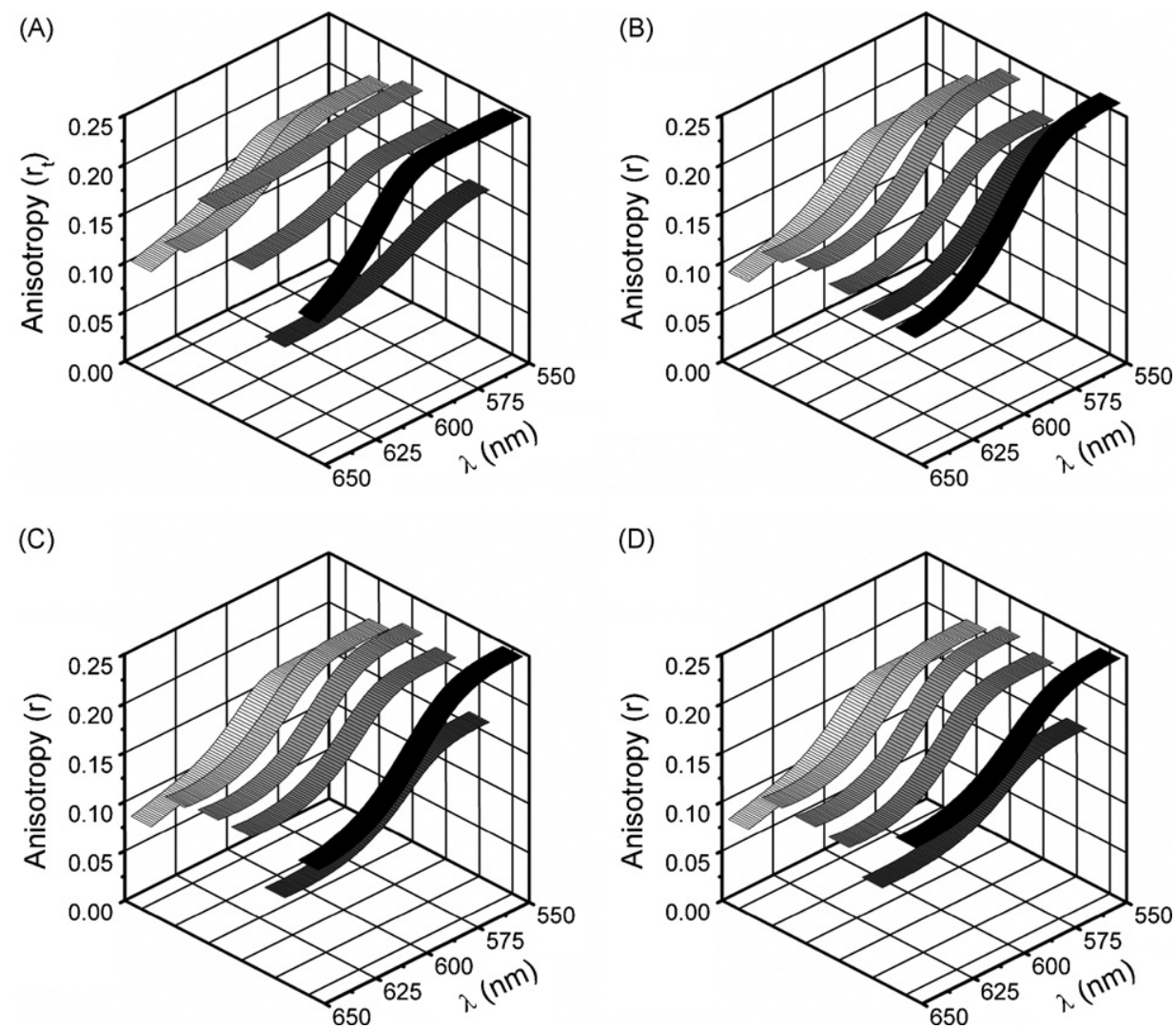

Fig. 8. Variation of theoretical fluorescence anisotropies $\left(r_{t}\right)$ of Nile Red for the systems DODAB

], DODAB $\left(60^{\circ}\right)$

], DODAB:MO $(2: 1)$

], DODAB:MO (1:1)

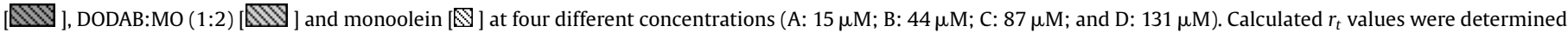
through the two state model previously described (Eq. (2)). 

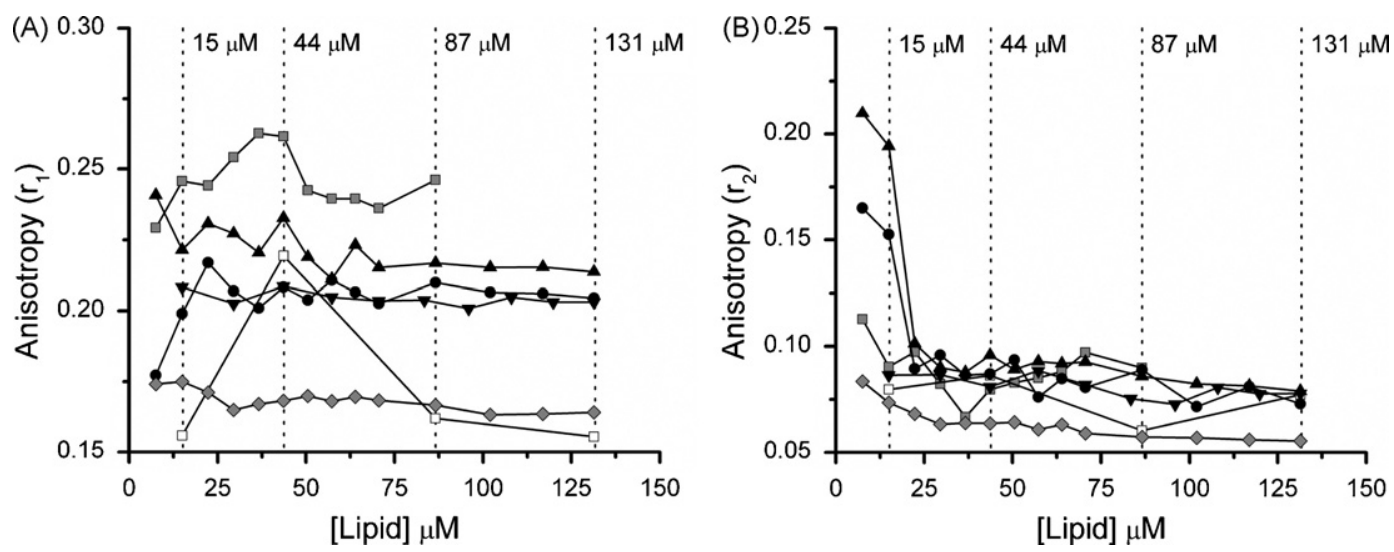

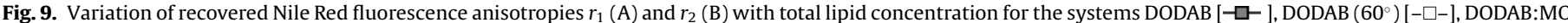

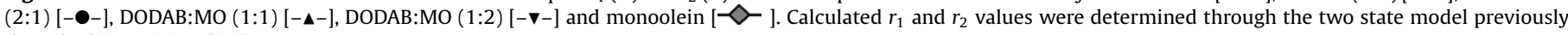
described (Eqs. (7) and (8)).
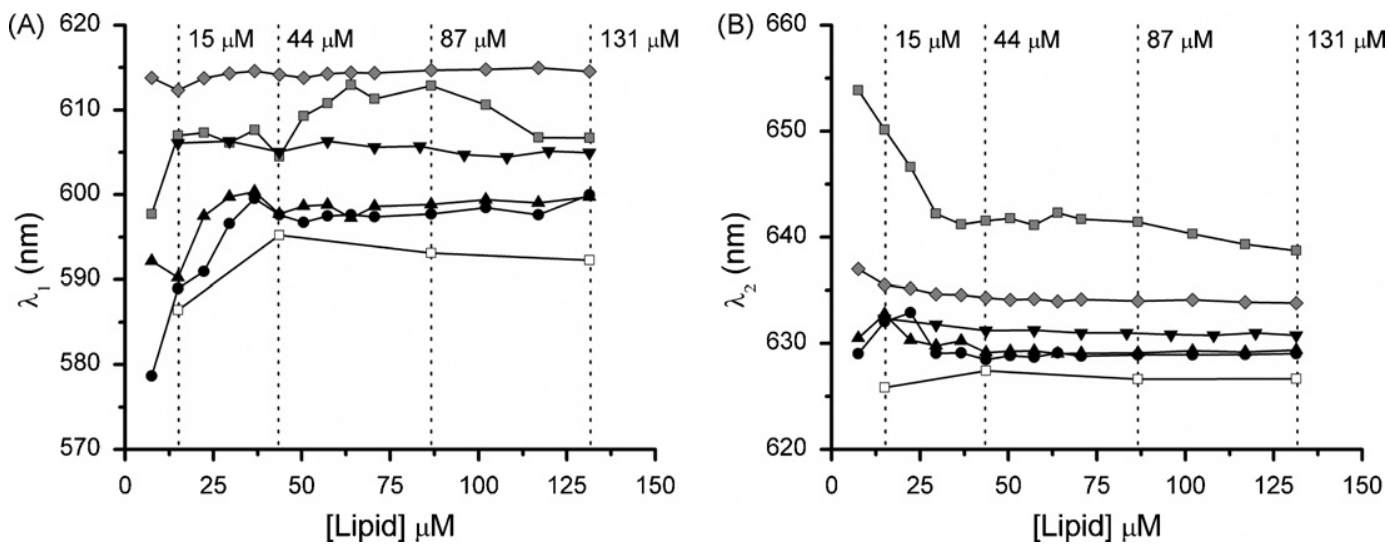

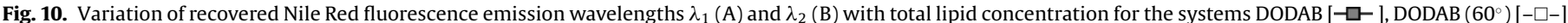

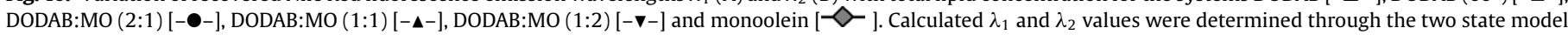
previously described.

Scattering $\left(90^{\circ} \mathrm{SLS}\right)$. The observed changes markedly depend on the MO content. Nile Red and $90^{\circ}$ SLS report common variations only up to the maximum change of size/shape of the lipid aggregates. As shape variations are not expected to cause 15 times increase in light scattering intensity, we think that other type of lipid aggregates are formed that upon reaching a critical concentration (at the

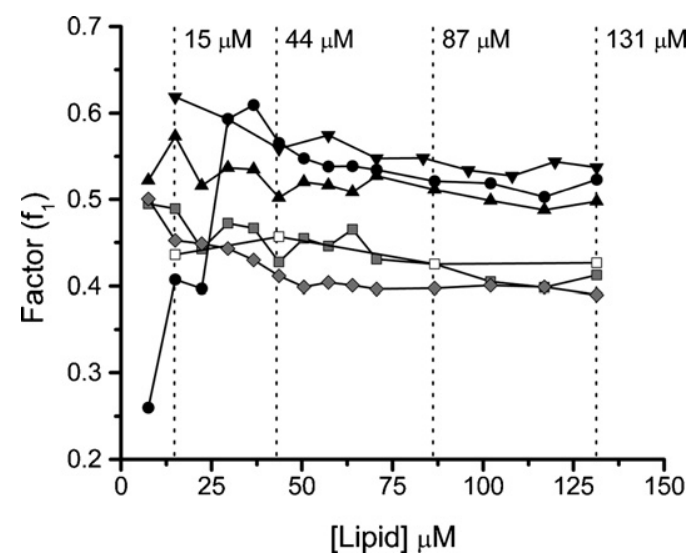

Fig. 11. Variation of recovered Nile Red fluorescence distribution factor $\left(f_{1}\right)$ with total lipid concentration for the systems DODAB $[-\square-]$, DODAB $\left(60^{\circ}\right)[-\square-]$,

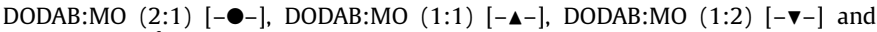
monoolein $\left[\checkmark\right.$ ]. Calculated $f_{1}$ values were determined through the two state model previously described (Eq. (9)). $90^{\circ}$ SLS maximum) reorganize themselves into the starting vesicular population. In the case of pure DODAB, these other aggregates can be bilayer fragments as already observed for sonicated lipid dispersions [12]. In the DODAB:MO mixed systems, the additional aggregate population can have a different MO content and acquire very different structures according to its tendency to form structures with negative curvature. These can be, for example, cubic bicontinuous structures.

\section{Acknowledgements}

The authors would like to thank to FCT-Portugal for funding through Centro de Física of University of Minho and through project PTDC/QUI/69795/2006.

\section{References}

[1] P.R. Cullis, B. deKruijff, Lipid polymorphism and the functional roles of lipids in biological membranes, Biochimica et Biophysica Acta 559 (1979) 399-420.

[2] S. Hui, A. Sen, Effects of lipid packing on polymorphic phase behavior and membrane properties, Proceedings of the National Academy of Sciences of the United States of America 86 (1989) 5825-5829.

[3] V. Luzzati, F. Husson, The structure of liquid crystalline phases of lipid-water systems, Journal of Cell Biology 12 (1962) 207-219.

[4] S.M. Gruner, Lipid polymorphism-the molecular basis of nonbilayer phases, Annual Reviews of Biophysics and Biophysical Chemistry 14 (1985) 211-238.

[5] P.R. Cullis, M.J. Hope, C.P.S. Tilcock, Lipid polymorphism and the roles of lipids in membranes, Chemistry and Physics of Lipids 40 (1986) 127-144.

[6] D. Needham, R.S. Nunn, Elastic deformation and failure of lipid bilayer membranes, Biophysical Journal 58 (1990) 997-1009. 
[7] D.M. Anderson, S.M. Gruner, S. Leibler, Geometrical aspects of the frustration in the cubic phases of lyotropic liquid crystals, Proceedings of the National Academy of Sciences of the United States of America 85 (1988) 5364-5368.

[8] R.P. Rand, N.L. Fuller, S.M. Gruner, V.A. Parsegian, Membrane curvature, lipid segregation, and structural transitions for phospholipids under dual-solvent stress, Biochemistry 29 (1990) 76-87.

[9] M.B. Sankaram, T.E. Thompson, Cholesterol-induced fluid-phase immiscibility in membranes, Proceedings of the National Academy of Sciences of the United States of America 88 (1991) 8686-8690.

[10] R.H. Templer, B.J. Khoo, J.M. Seddon, Gaussian curvature modulus of an amphiphilic monolayer, Langmuir 14 (1998) 7427-7434.

[11] A.M.C. Ribeiro, Synthetic amphiphile vesicles, Chemical Society Reviews 21 (1992) 209.

[12] E. Feitosa, W. Brown, Fragment and vesicle structures in sonicated dispersions of dioctadecyldimethylammonium bromide, Langmuir 13 (1997) 4810 4816.

[13] E. Feitosa, P.C.A. Barreleiro, G. Olofsson, Phase transition in dioctadecyldimethylammonium bromide and chloride vesicles prepared by different methods, Chemistry and Physics of Lipids 105 (2000) 201-213.

[14] C.R. Benatti, E. Feitosa, R.M. Fernandez, M. Lamy-Freund, Structural and thermal characterization of dioctadecyldimethylammonium bromide dispersions by spin labels, Chemistry and Physics of Lipids 111 (2001) 93.

[15] E. Feitosa, G. Karlsson, K. Edwards, Unilamellar vesicles obtained by simply mixing dioctadecyldimethylammonium chloride and bromide with water, Chemistry and Physics of Lipids 140 (2006) 66

[16] A.M.C. Ribeiro, Lipid bilayer fragments and disks in drug delivery, Current Medicinal Chemistry 13 (2006) 1359-1370.

[17] B. Geil, T. Feiweier, E.-M. Pospiech, J. Eisenblätter, Relating structure and translational dynamics in aqueous dispersions of monoolein, Chemistry and Physics of Lipids 106 (2000) 115-126.

[18] V. Luzzati, Biological significance of lipid polymorphism-the cubic phases, Current Opinion in Structural Biology 7 (1997) 661-668.

[19] L.M.R. Singh, NMR Studies of Membrane-Binding Peptides in Monoolein Cubic Phases, Department of Molecular Biology and Biochemistry, Simon Fraser University, Burnaby (Canada), 2005, p. 240.

[20] R.M. Epand, Lipid polymorphism and protein-lipid interactions, Biochimica et Biophysica Acta 1376 (1998) 353-368.

[21] R. Lipowsky, E. Sackmann, Structure and Dynamics of Membranes, 2nd ed., N. Holland-Elsevier, Amsterdam (Netherlands), 2004, p. 1036

[22] V. Chupin, J.A. Killian, B. deKruijff, Effect of phospholipids and a transmembrane peptide on the stability of the cubic phase of monoolein implication for protein crystallization from a cubic phase, Biophysical Journal 84 (2003) 2373 2381.

[23] S.M. Masum, S.J. Li, Y. Tamba, Y. Yamashita, M.T. Tanaka, Effect of de novo designed peptides interacting with the lipid-membrane interface on the stability of the cubic phases of the monoolein membrane, Langmuir 19 (2003) 4745-4753.
[24] E.M. Landau, J.P. Rosenbusch, Lipidic cubic phases-a novel concept for the crystallization of membrane proteins, Proceedings of the National Academy of Sciences of the United States of America 93 (1996) 14532-14535.

[25] M. Caffrey, A lipid's eye view of membrane protein crystallization in mesophases, Current Opinion in Structural Biology 10 (2000) 486-497.

[26] M. Caffrey, Membrane protein crystallization, Journal of Structural Biology 142 (2003) 108-132.

[27] C. Sennoga, A. Heron, J.M. Seddon, R.H. Templer, B. Hankamer, Membraneprotein crystallization-temperature-dependent phase behaviour of monoolein-detergent mixtures, Acta Crystallographica Section D 59 (2003) 239-246.

[28] N. Carlsson, S. Nima, V. Marina, A. Björn, Bicontinuous cubic phase of monoolein and water as medium for electrophoresis of both membrane-bound probes and DNA, Langmuir 22 (2006) 4408-4414.

[29] J.P.N. Silva, P.J.G. Coutinho, M.E.C.D.R. Oliveira, Characterization of monooleinbased lipoplexes using fluorescence spectroscopy, Journal of Fluorescence 18 (2008) 555-562.

[30] S.J. Li, Y. Yamashita, M. Yamazaki, Effect of electrostatic interactions on phase stability of cubic phases of membranes of monoolein-dioleoylphosphatidic acid mixtures, Biophysical Journal 81 (2001) 983-993.31.

[31] Y. Okamoto, S.M. Masum, H. Miyazawa, M. Yamazaki, Low-pH-induced transformation of bilayer membrane into bicontinuous cubic phase in dioleoylphosphatidylserine-monoolein membranes, Langmuir 24 (2008) 3400-3406.

[32] Y. Aota-Nakano, S.J. Li, M. Yamazaki, Effects of electrostatic interaction on the phase stability and structures of cubic phases of monoolein-oleic acid mixture membranes, Biochimica et Biophysica Acta 1461 (1999) 96-102.

[33] T.S. Awad, Y. Okamoto, S.M. Masum, M. Yamazaki, Formation of cubic phases from large unilamellar vesicles of dioleoylphosphatidylglycerol monoolein membranes induced by low concentrations of $\mathrm{Ca}^{2+}$, Langmuir 21 (2005) 11556-11561.

[34] S.M. Masum, S.J. Li, T.S. Awad, M. Yamazaki, Effect of positively charged short peptides on stability of cubic phases of monoolein-dioleoylphosphatidic acid mixtures, Langmuir 21 (2005) 5290-5297.

[35] G. Hungerford, E.M.S. Castanheira, A.L.F. Baptista, P.J.G. Coutinho, M.E.C.D. Real Oliveira, Domain formation in DODAB-cholesterol mixed systems monitored via Nile Red anisotropy, Journal of Fluorescence 15 (2005) 835-840.

[36] G.C. Shearman, O. Ces, R.H. Templer, J.M. Seddon, Inverse lyotropic phases of lipids and membrane curvature, Journal of Physics: Condensed Matter 18 (2006) S1105-S1124.

[37] P.J.G. Coutinho, E.M.S. Castanheira, M. Ceu Rei, M. Elisabete, C.D. Real Oliveira, Nile Red and DCM fluorescence anisotropy studies in C12E7DPPC mixed systems, Journal of Physical Chemistry B 106 (2002) 12841-12846.

[38] M.M.G. Krishna, Excited-state kinetics of the hydrophobic probe Nile Red in membranes and micelles, Journal of Physical Chemistry A 103 (1999) 3589-3595.

[39] A. Cser, K. Nagy, L. Biczok, Fluorescence lifetime of Nile Red as a probe for the hydrogen bonding strength with its microenvironment, Chemical Physics Letters 360 (2002) 473-478. 